\title{
Editorial
}

\section{Obstetrical epidural anaesthesia in remote hospitals}

In this issue of the Journal, Dr. Orser describes the experience with obstetrical epidural anaesthesia in a small ( 40 beds), remote Canadian Hospital staffed by family practitioners.' Over a ten year period, seven per cent of obstetric patients received an epidural anaesthetic administered by one of nine different anaesthetists who had six months or less of formal anaesthesia training. Dr. Orser is to be commended for a thorough review of the experience and for addressing the issues surrounding the use of epidural anaesthesia within small obstetric units. The hospital which formed the basis of this report is in Newfoundland, but the problem of providing medical care in remote communities is common to all Canadian Provinces and Territories. In the Province of Manitoba, for example, rural medical services are provided in 70 hospitals, 50 of which have less than 30 beds. Many of these hospitals are inaccessible by road for all or part of the year. Fully 15 per cent of all obstetrical deliveries in the province are performed at hospitals with less than 50 beds and where centralization of care is impossible except at prohibitive cost. Our task is to ensure that the best obstetric anaesthesia care is made available at these hospitals.

The two main issues arising from Orser's report and general consideration of the geographical distribution of obstetric care in Canada are:

1 Should epidural anaesthesia be provided in low volume obstetric units with neither specialist anaesthetists or specialist obstetricians? and

2 What standards should be applied with respect to obstetrical epidural anaesthesia in those institutions?

The first question is more difficult to answer than the second. Orser reports complication rates which compare favourably with those published from a much larger teaching unit. ${ }^{2}$ The failure rate of epidural anaesthesia for

Department of Anaesthesia, Universily of Manitoba, 3rd Floor Lennox Bell Lodge, Health Sciences Centre, Winnjpeg, Manitoba; R3E 1 X2
Caesarean section was, however much higher in her report (32 vs three per cent). Teaching physicians to establish an epidural is not difficult. Maintaining that expertise in the face of a low volume of experience (average of one epidural anaesthetic per month) is far more difficult. Equally important is the maintenance of an entire obstetric team with the skill and experience to manage patients with epidural anaesthetics. This includes, in addition to the anaesthetist, the obstetricianfamily practitioner, labour and delivery nurses, and recovery room nurses. One case per month is clearly insufficient to ensure adequate management of these abstetric patients. What frequency of cases would be necessary is impossible to state because of the lack of information of this type in the literature.

Epidural anaesthesia should be available even in small obstetric units, provided sufficient cases are managed to maintain the necessary skills and experience. If epidural anaesthesia was not available to these 116 patients at Baie Verte Hospital, the alternatives (heavy sedation, local perineal blocks and/or general anaesthetics) may have, in fact, been less than satisfactory alternatives.

Concerning standards, there is little doubt that no matter how few deliveries are performed, patients in remote hospitals deserve the same standards of care as patients in urban hospitals. This would suggest that the Canadian Anaesthetists' Society guidelines ${ }^{3}$ as documented by Orser be followed. Monitoring equipment must be available and used appropriately. Complications must be recognized early and managed adequately. The obstetrician-family practitioner must be prepared to perform forceps or vacuum deliveries (54 per cent of patients with epidurals in the present series). As well, the family practitioner-anaesthetist must be prepared to stay in the hospital throughout the duration of the epidural anaesthetic. Anything less is a compromise of standards and less than optimum patient care.

The training requirement for family practitioneranaesthetists is currently being addressed by several organizations in Canada, and is beyond the scope of this editorial. Suffice it to state that Canadian University 
Departments of Anaesthcsia agree that i minimum of one year of anaesthesia training is necessary.*

To summarize, Orser's article re-emphasizes that epidural anaesthesia can be safely provided in small and remote obstetric units. Such provision of anaesthetic services is to be encouraged, provided that adequate experience can be maintained, standards of care are not compromised, and appropriate quality assurance procedures are followed.

*Association of Canadian University Departments of Anaesthesia. Resolution passed at annual meeting, Calgary, Alberta, June 13, 1987.

\section{L'anesthésie épidurale obstétricale dans les hôpitaux des regions éloignées}

Docteur Orser, dans ce numéro du Joumal, décrit l'experience en amesthésie épidurale obstétricale dans un petit hôpital (40 lits), d'une région éloignée où les soins sont fournis par des médecins de farmille.' Pour une période de dix ans, sept pour cent des patientes en obstétrique ont reçu une anesthésie épidurale administrée par un des neuf praticiens généraux qui ont eu six mois ou moins d'entrainement en anesthésie. Docteur Orser doit être félicité pour cette revue extensive qui s'attaque alx problèmes entourant l'utilisation de l'anesthésie épidurale dans une petite unité obstétricale. L'hôpital en question est à Terre-Neuve et le problème des sains médicaux dans une région éloignée est commun à toutes les provinces et territoires canadiens. Au Manitoba par exemple les services médicaux ruraux sont foumis dans 77 hôpitaux dont 50 ayant moins que 30 lits. Plusieurs de ces hôpitaux sont inaccessibles par voie de terre pour toute ou une partie de l'année. Quinze pour cent des accouchements de la province ont lieu dans des hôpitaux ayant moins que 50 lits etou la centralisation des soins est impossible sauf à des coûts prohibitifs. Notre rôle est d'assurer que la meilleure anesthésie obstétricale soit disponible dans ces hôpitaux.

Les deux points majeurs qui ressortent du rapport du docteur Orser et des consultations générales sur la distribution géographique des sains obstétricaux au Canada sont:
1 L'anesthésie épidurale doit-elle être offerte dans des petites unités obstétricales en l'absenced'un spécialiste en anesthésie ou en obstếtrique, et

2 Quelles sont les normes qui doivent être appliquées à la pratique de l'anesthésie épidurale obstétricale dans ces institutions?

La première question est plus difficile à répondre que la seconde. D'après le rapport d'Orser le taux de complication se compare favorablement à celui publié dans des centres plus grands d'enseignement. ${ }^{2}$ Le taux d'échec de l'anesthésie épidurale pour une césurienne est cependant plus haut dans un rapport (32 vs trois pour cent). Apprendre aux médecins à faire une épidurale n'est pas difficile. Maintenir cette expertise avec un volume faible (en moyenne une épidurale par mois) est beaucoup plus difficile. L'ćgale importance est le maintien d'une équipe obstétricale possédant l'expérience et l'habileté pour prendre soin des patientes devant subir une épidurale. Ceci inclut en plus d'un anesthésiste, l'obstétricienmédecin de famille, les infirmières pour le travail et l'accouchement et les infirmières pour la salle de réveil. Un cas par mois est définitivement insuffisant pour assurer des soins adéquats pour ce groupe de patientes. Le nombre de cas nécessaire est impossible à déterminer par manque d'information sur ce sujet dans la littérature.

L'anesthésie épiđurale doit être disponible même dans des petites unités obstétricales à condition d'avoir assez de cas pour maintenir une habileté et une expertise acceptable. Si l'anesthésie épidurale n'était pas disponible à ces 116 patientes à l'Hôpital de la Bate Verte, les alternatives (forte sédation, bloc périnéal local, et/ou anesthèsie générale) auraient pu en fait être des alternatives moins satisfaisantcs.

Concemant les normes, il y a aucun doute qu'indépendamment du nombre d'accouchements les patientes dans ces hôpitaux éloignés mériteraient les mềmes normes de soin que celles des centres urbains. Ceci suggère que les directives de la Sociélé Canadienne d ${ }^{1}$ Anesthésie, ${ }^{3}$ comme le suggère docteur Orser, doivent être suivies. L'équipement de monitorage doit être disponible et utilisé adéquatement. Les complications doivent être reconnues précocément et traitées adéquatement. L'obstétricienmédecin de famille doit être préparé à appliquer des forceps ou accomplir des accouchements par ventouse (54 pour cent des patientes ayant subi une épidurale dans cette série). Ainsi l'anesthésiste-praticien général dojt être préparé à demeurer à l'hôpital tout au cours de la durée de l'anesthésie épidurale. Des compromis concernant ces points seraient des compromis sur les normes de pratique et seraient inacceptables pour les soins des patientes.

L'entraînement nécessaire pour le praticien généralanesthésiste est actuellement en train d'être évalué par plusieurs organisations au Canada et n'est pas le sujet de 
cet éditorial. Il suffit de dire que les départements d'anesthésie des universités canadiennes admettent qu'un minimum d'un an d'anesthésie est nécessairc.

En résumé, l'article d'Orser remet l'emphase sur le fait que l'anesthésie Épidurale peut être administrée avcc sécurité dans une petite unité obstétricale en région éloignée. Foumir de tels services est à encourager à condition d'avoir et de maintenir l'expertise adéquate et de ne pas compromettre les normes de soin et de suivre les procédures appropriées pour assurer des soins de qualité.

\section{References}

1 Orser B. Obstetrical epidural anaesthesia in a Canadian outpost hospital. Can J Anaesth 1988: 35: 503-6.

2 Ong B, Cohen MM, Cumming M, Paiahniuk RJ. Obstetrical anacsthesia at Winnipeg Women's Hospital 1975-83: Anaesthetic techniques and complications. Can J Anaesth 1987; 34: 294-9.

3 Guidelines to the practice of anaesthesia. Canadian Anaesthetists' Sociery Newsletter. Canadian Anaesthetists' Society, 187 Gerrard Street East, Toronto, Ontario, M5A 2E5. Vol 2: No. 3: Sept 1986. 